\title{
Clinical Use of Growth Factors in the Improvement of Skin Wound Healing
}

\author{
Sarita Mac Cornick, Silvana Aparecida Alves Corrêa de Noronha, Verônica Chominski, \\ Samuel Marcos Ribeiro de Noronha, Lydia Masako Ferreira, Alfredo Gragnani*
}

Division of Plastic Surgery, Department of Surgery, Federal University of Sao Paulo, Paulista School of Medicine (UNIFESP-EPM), Sao Paulo, Brazil

Email: ${ }^{*}$ alfredogf@ig.com.br

Received 9 September 2014; revised 9 October 2014; accepted 9 November 2014

Copyright (C) 2014 by authors and Scientific Research Publishing Inc.

This work is licensed under the Creative Commons Attribution International License (CC BY). http://creativecommons.org/licenses/by/4.0/

(c) $\underset{\mathrm{EY}}{\mathrm{F}}$ Open Access

\section{Abstract}

To discuss the normal mechanism of wound healing (WH), the role of growth factors (GF) in preclinical and clinical studies and its importance in the healing of abnormal wound therapy. For search, we used the PUBMED and LILACS database, and the following descriptors: skin, wound healing, growth factor and clinical trials. We also prioritized the analysis of the Clinical Trials in the previous 10 years. Although there are many studies being conducted in the pre-clinical phase, we see that there are few studies in the clinical phase. 274 studies were identified, and 58 were selected. After tissue injury, repair occurs through coordinated biological actions that are healing. The importance of the study of healing in the skin is not only because of its anatomical location, easy access and exposure, but also because of its vital function. There is accumulating evidence that the process of healing after injury may be mediated by several GF. However, may this class of molecules even act effectively on the clinical response of this pathological condition? Many preclinical studies (in vitro and in vivo) reinforce the importance and efficacy of GF in the regeneration of damaged skin. Furthermore, recent studies have reported the use in adjuvant or not, of GF in clinical treatment to improve WH in humans. Therefore, we conclude that it seems to be effective by the use of GF in adjuvant or not in WH. However, it still seems to be necessary to carry out more clinical trials in phase I and II.

\section{Keywords}

Growth Factors, Wound Healing, Wound and Injuries, Keloid, Skin

\section{Introduction}

The ability to restore normality and continuity of the injured skin is species-specific. Scars and abnormalities

\footnotetext{
${ }^{*}$ Corresponding author.
}

How to cite this paper: Cornick, S.M., de Noronha, S.A.A.C., Chominski, V., de Noronha, S.M.R., Ferreira, L.M. and Gragnani, A. (2014) Clinical Use of Growth Factors in the Improvement of Skin Wound Healing. Open Journal of Clinical Diagnostics, 4, 227-236. http://dx.doi.org/10.4236/ojcd.2014.44032 
associated with a poor skin restoration not only prevent normal function of the organ, but also represent major challenges in clinical management. The damage to the protective layer can be devastating for both the patient and for society that attends. In 2004, almost 11 million cases of burns [1] and 7 million chronic skin ulcers caused by high blood pressure, or venous insufficiency and diabetes mellitus each year in the US alone are affected by abnormal healing. This translates in costs that have been estimated at $\$ 25$ billion annually in the United States, in an attempt to reduce severe disability and death resulting from such severe skin lesions [2]. The cascade of normal wound healing consists of three phases and their components (growth factors, cytokines and inflammatory cells) appear to play a role in the regeneration of abnormal lesions [3].

Here, we will give special attention to one of these components, growth factors. We begin with a description of each phase of the healing process, and then present (in vitro and in vivo) preclinical studies that have demonstrated the importance of these factors in wound healing. And, finally, we present the research being conducted in the clinical phase that attests to the efficacy of growth factors in the regeneration of skin wounds in humans.

\section{Methods}

Scientific papers were studied regarding our model and methodological approach for the period from January 2004 up until August 2014. The research was performed using PubMed database. The main keywords used were "growth factors, wound healing and skin". This was done in accordance with the DeCS/MeSH version and by also crossing information using the keyword "and". By applying such norms and procedures, 274 papers were identified. But to create this paper we have used 58, because many articles were related with the healing of other tissues than the skin. The research was organized according to the methodology of how is the stages of healing and after the application in preclinical and in clinical phases experiments. Moreover, we have prioritized works related to the skin and literature reviews.

\section{Results}

\subsection{Wound Healing}

Normal wound healing is a dynamic series of events involving the coordinated blood cells, proteins, growth factors and proteases components of the extracellular matrix interaction. The process of wound healing can be divided into: 1) the inflammatory phase; 2) proliferation phase; and 3) maturation phase [3].

\subsubsection{Inflammatory Phase}

The inflammatory phase is the first of wound healing and is characterized by the hemostasis and by the inflammation. Hemostasis is initiated during exposure of collagen during the formation of the wound, which activates the cascade of intrinsic and extrinsic coagulation. Moreover, tissue damage causes the release of thromboxane A2 and prostaglandin 2-alpha to the wound bed causing a potent vasoconstrictor response. Therefore, the leakage of blood constituents provides for the formation of the blood clot enhancing the initial hemostatic response. This helps limit the bleeding and provides an initial extracellular matrix for cell migration [3].

Platelets are known for their role in homeostasis where they help prevent blood loss at sites of vascular injury. To do this, they adhere, aggregate and form a procoagulant surface leading to thrombin generation and fibrin formation. Platelets also release substances that promote tissue repair and influence the reactivity of vascular and other blood cells in angiogenesis and inflammation. They contain storage pools of growth factors including PDGF, TGF-beta and VEGF [4].

After hemostasis is achieved capillary, vasodilation results in secondary release to local histamine by local activation of the complement cascade leak. The increased blood flow and vascular permeability changes allow the migration of inflammatory cells into the wound bed. The presence of foreign bodies further stimulates activation of the alternative pathway of complement. The activation of the complement cascade activates C3 results in cleavage of non-enzymatic protein and interactions that eventually stimulate inflammatory cells and lysis of bacteria [3].

After complement activation and recruitment of platelets, neutrophils migrate to the wound. This cell is responsible for waste disposal by complement mediated by lysis of foreign organisms, the bacterial opsonization and destruction through mechanisms of oxidative stress (i.e., the formation of superoxide and hydrogen peroxide). Neutrophils kill bacteria and decontaminate the wound of foreign debris. These wastes are then extruded 
with the scar or phagocyted by macrophages. Macrophages are important phagocytic cells that play a key role in wound healing [5]. Besides direct phagocytosis of bacteria and foreign material, numerous macrophages secrete enzymes and cytokines, collagenases that debride wounds; interleukins and tumor necrosis factor (TNF) that stimulate fibroblasts and promote angiogenesis, and TGF-beta1, FGF-7, FGF-2 and AGF that were chemotactic to keratinocytes [6]. Macrophages also secrete PDGF and vascular endothelial growth factor (VEGF), which initiate the formation of inflammation granulation tissue, Reepithelialization Matrix formation and remodeling [7].

\subsubsection{Proliferation Phase}

The proliferative phase is marked by epithelialization, by angiogenesis, by the formation of granulation tissue, and by the collagen deposition. The epithelialization occurs within hours after injury in the repair of wounds. With the intact basement membrane, epithelial cells migrate above the normal range as in a skin burn first degree by which epithelial progenitor cells remain intact below the wound, and the normal layers in the epidermis are restored in 2 - 3 days. If the basement membrane has been damaged, as in deep burn, then the normal epidermal cells of skin appendages (e.g., hair follicles, sweat glands) and of periphery of the wound reepithelize [3].

Neovascularization is required for providing nutrients to the wound and help maintain the granulation tissue bed. Angiogenesis has been attributed to various molecules, including fibroblast growth factor (FGF), VEGF, TGF-beta, angiogenin, the angiotropina, the angiopoietin-1 to tumor necrosis factor alpha (TNF-alfa) and thrombospondin [8] [9]. In different clinical conditions such as diabetes and vascular disease, this supply critical of nutrient through capillaries is insufficient to maintain tissue deposition in the granulation step, therefore, in a chronically unhealed wound.

The proliferative phase ends with granulation tissue formation. This new stroma begins to invade the wound space close to four days after injury. The new blood vessels at this time have provided a facilitated entry point into the wound to cells such as macrophages and fibroblasts [3].

Macrophages still provide more growth factors that stimulate angiogenesis and fibroplasia. The secreted PDGF [7] and TGF-beta [10] [11] along with extracellular matrix molecules stimulate the differentiation of fibroblasts to produce ground substance and then collagen. Fibroblasts are the major players of the synthesis, deposition, and remodeling of the extracellular matrix providing strength and substance to the wound.

\subsubsection{Maturation Phase}

The third and final phase of wound healing is the maturation phase. This transition is characterized by granulation to form scar tissue. This phase ends two weeks after the injury. The wound undergoes contraction, resulting in a smaller amount of apparent scar tissue. The deposition of collagen by fibroblasts continues for a long period with a large increase occurring after three weeks after tissue injury [3].

The entire process is a dynamic continuum dictated by numerous factors and growth of cells with an overlap of each of the three phases of wound healing to provide a continuous remodeling [12].

\subsection{Growth Factors and Their Implication in Wound Healing: Pre-Clinical Trials}

In the previous section, we describe the normal wound healing, in that these growth factors influence many processes including proliferation and migration of various types of cells, chemotaxis of inflammatory cells and fibroblasts. The coagulation cascade is the first mechanism activated in the wound-healing process, and activated platelets release pro-fibrotic factors like PDGF and TGF-beta 1 [13].

The sequential and orderly progression through the stages of wound healing requires a number of different cell, humoral factors and other agents to interact so that wound closure occurs properly and on time. Keloids and hypertrophic scars are suboptimal consequences of skin wound healing, and are believed to be unique to human skin. These two clinical entities belong to a spectrum of fibro proliferative disorders, and are difficult to differentiate histologically in the absence of relevant clinical details [14]. Below we report some active growth factors in wound healing in pathological situations that were evaluated in pre-clinical research process.

PDGF is a key mediator in wound healing, and its importance is highlighted by being the first recombinant growth factor approved for topical application to accelerate wound closure [15] [16]. At baseline levels, platelets function as a natural reservoir for growth factors including platelet-derived growth factor (PDGF), epidermal growth factor (EGF), transforming growth factor-beta 1 (TGF-beta 1), vascular endothelial growth 
factor (VEGF), basic fibroblast growth factor (FGF), hepatocyte growth factor (HGF), and insulin-like growth factor (IGF-I), to name a few [17] [18]. Such growth factors are released from the alpha granules of activated platelets and are involved in important cellular processes including mitogenesis, chemotaxis, differentiation, and metabolism [18].

FGF is a diverse family of mitogens that affect a large number of cell types, with important implications for wound healing [16] [19]. In wounds, the expression of FGF detected by immunohistochemical methods is greater in the center of the wound with progressive decline in intensity toward the periphery and almost none found in uninjured skin [20]. The acid fibroblast growth factor (aFGF) and basic (bFGF) have been identified in wound fluid, especially in the early stages of healing [16]. In human amniotic fluids, cytokines such as PDGF and bFGF seem to stimulate the cutaneous fibroblast proliferation [21]. The role of bFGF in wound healing has been demonstrated in vitro and in vivo through their actions in the activation of local macrophages, increasing the production of extracellular matrix components, proliferation and differentiation of neuroectodermal and mesodermal derivatives, fibroblast proliferation, proliferation and migration of endothelial cells for angiogenesis and reepitelialização [22].

The most extensive experience with clinical trials of growth factor in the healing wound therapy has been with bFGF [16]. The CFGF similar to bFGF in their biological properties also influence the proliferation of fibroblasts and endothelial cells, promoting wound healing and angiogenesis [23]. The keratinocyte growth factor 1 (KGF-1), another member of the FGF family, promotes epithelialization through its effects on the proliferation and differentiation of epithelial cells, while inhibiting apoptosis [24]. The keratinocyte growth factor 2 (KGF-2) similarly affects reepithelialization but also stimulates the formation of granulation tissue and collagen deposition in wound healing [16].

KGF stimulated wound closure in cultured monolayers primarily by increasing cell spreading and migration at the wound edge, and increased cellular adhesion in an EGFR activation-dependent manner [25]. Using a tracheal transplant model in mice, Gomperts and colleagues [26] showed that KGF accelerated the repair of injured tracheal epithelium.

The EGFs comprise another family of mitogens that appears to be present in the wound fluid and has significant effects on healing [19] [27]-[29]. EGF is mitogenic for epithelial, mesothelial, and endothelial cells and has been shown to accelerate re-epithelialization, increase proliferation and tensile strength of healed dermis, and improve overall wound healing [27] [28]. As for healing burns, however, there is conflicting evidence on the presence of EGF in wound fluid [16] [19]. Two other members of the EGF family, epidermal growth factor binding heparin (HB-EGF) and TGF-alfa have been found in significant levels in burn wound fluid [19]. Evidence for the role of HB-EGF and TGF-alfa in wound healing is seen through its effects on the stimulation of keratinocyte proliferation and to accelerate the reepithelialization [30]. HB -EGF is also a mitogen for fibroblasts and acts on the stimulation of the formation of granulation tissue [19].

The vascular endothelial growth factors (VEGF) comprise a family of cytokine growth factors identified as important mediators of angiogenesis, lymphangiogenesis and vascular permeability [31]. Recent evidence suggests that proangiogenic effects of VEGF may be mediated through stimulation of the synthesis of hydrogen sulfide from endothelial cells, promoting their proliferation, migration and permeability [32]. There are also data to support the role of VEGF in stimulating epithelialization and collagen deposition [32].

It has been theorized that reductions in VEGF lead to down regulation of nephrin expression, subsequently resulting in podocyte injury and proteinuria [33]. Several studies have demonstrated the benefits of exogenous VEGF therapy in the acceleration and improving healing by topical application of amplified expression and gene transfer of growth factor in adenoviral vector [34]-[36].

Conversely, the neutralizing antibody to VEGF significantly reduces a buildup of fluid in the wound, the formation of granulation tissue and angiogenesis [31]. Two other members of the VEGF, VEGF-C and VEGF-D, family are involved in the regulation of angiogenesis but not linfoangiogenesis [19]. Although VEGF seems to be beneficial in promoting wound healing for burns, a caveat to this evidence suggests that increased serum VEGF is responsible for edema, and the complications associated with swelling after injury by burning [31].

The insulin-like factors I and II (IGF-I and IGF-II) growth are well described as anti-catabolic and pro-anabolic agents that act in a multitude of cell types systematically [19]. IGF-I is in fluid burn wounds as part of the normal response to injury and the expression of IGF-I and IGF-II have been shown to be significantly over-regulated in the wound healed epidermal cells that normally express minor amounts the protein product [19].

It has been demonstrated that nerve growth factor (NGF) affecting various aspects of wound healing [19]. 
Given the variety of neurogenic involvement and in wound healing mediated processes, the growth of cutaneous nerve and the restoration of normal innervation, regulated by NGF is essential for the normal healing process [37]. Sensory nerves are responsible for the release of substance P, a neuropeptide associated with stimulation of epithelial cell proliferation and migration and acceleration of wound healing in a murine model when applied topically [37]. NGF also appears to activate cell proliferation and inhibit apoptosis in keratinocytes and endothelial cells and promote the migration of fibroblasts [38].

The TGF-beta family consists of many members, with a great diversity in their actions [39]. Of particular interest to the healing of wounds, TGF-beta 1, TGF-beta 2 and TGF-beta 3 isoforms share a number of functions such as the stimulation of proliferation and production of fibroblasts, extracellular matrix components and cell adhesion molecules, whereas inhibits keratinocyte and most other cell types [16] .

Accordingly, mice deficient in TGF-beta 1 seems to have poor wound healing with impaired formation of granulation, the production of extracellular matrix tissue and re-epithelialization [39]. Interestingly, another study found that deficiency of TGF-beta 1 was associated with delayed healing of wounds only TGF-beta 2 and TGF-beta 3 were not expressed [40]. However, the recent evidence suggesting that TGF-beta 1 and TGF-beta 2 negatively influences the healing of wounds through the delay epithelialization and increased scarring and contraction [41]. TGF-beta also serves as an anti-inflammatory agent, or any state of increased persistent inflammation such as infection and prolonged healing which can lead to overexpression of TGF-beta and subsequent formation of keloid or hypertrophic scarring [38]. By contrast, fetal wounds that are less prone skin scarring are associated with low levels of TGF-beta 1 and TGF-beta 2 [19] [42] [43].

Intradermal injection of neutralizing antibodies to TGF-beta 1 alone or TGF-beta 1 and TGF-beta 2 has been associated with reduced scar formation and wound contraction [44]. The use of a synthetic TGF-beta applied topically peptagonista showed acceleration of re-epithelialization, improves wound healing in general and decreased scarring and contracture of excisional and burn on rabbits excisional wound model in pigs [45].

Contrary to the adverse effects of TGF-beta 1 and TGF-beta 2, TGF-beta 3 appears to be beneficial in the improvement of wound healing [43]. Expression of TGF-beta 3 is elevated in fetal wounds compared with adult wounds, which are more prone to scarring [42]. Experimental models of disability and deletion of TGF-beta 3 showed delayed wound healing and increased scarring [43] [46]. Moreover, the therapeutic use of recombinant human TGF-beta 3 (avotermin, Juvista, Branch Group, Manchester, UK) showed accelerated wound healing and improvement in the appearance of cutaneous scar after surgery [43].

The macrophage colony-stimulating factor and granulocyte (GM-CSF) is a multipotential cytokine with many diverse functions [47] [48]. In the context of the burn, the benefit of GM-CSF is two times higher. It has positive effects on enhancing wound healing and acts also promoting and restoring a compromised immune response [48]. The hyper-regulation and production of this cytokine in keratinocytes, fibroblasts, endothelial cells, macrophages and dendritic cells occurs after tissue injury as part of the process of wound healing [16].

\subsection{Clinical Efficacy of Growth Factors in Skin Wound Healing}

As we saw earlier, some growth factors has shown efficacy in cutaneous wound healing by basic research in vitro or in animal models, but clinical efficacy has yet to be more consistent data to be proven.

Now our focus will be clinical trials conducted during the last ten years, in which we found that especially in skin ulcers, diabetic foot, deep burns and surgical wounds, in which the growth factors that are most active in improving the growth factors fibroblast, transforming beta, epidermal, vascular endothelial and platelet derived (FGF, TGF-beta, EGF, VEGF and PDGF).

Some of these studies have evaluated both the safety and the efficacy of the topical use of growth factor. YAO AND COLLEAGUES in 2006 studied recombinant basic fibroblast growth factor (rbFGF/ACS; recombinant basic fibroblast growth factor loaded on a kind of absorbable collagen sponge) that was added to a kind of absorbable collagen sponge in patients with chronic traumatic ulcers. This double-blind controlled study included 58 patients. Patients were randomized into two groups. After debridement, the wounds were covered with sponge containing the growth factor or connected with sterile gauze in control group. The sponge containing the growth factor significantly increased the incidence of complete wound closure, shortened the healing time and improved the quality of healing of chronic traumatic ulcers, then showing the efficacy of this growth factor in chronic wounds [49].

The effectiveness of topical treatment of the recombinant human acid fibroblast growth factors (rh-aFGF) was 
also evaluated in deep burn. To investigate its effectiveness in deep partial thickness burn or donor site skin graft a multicentre, double-blind, randomized, placebo-controlled study to assess the rate trial and healing time was drawn after applying rh-aFGF. Laboratory tests and abnormal signs were used to assess the toxic effects. The results showed that the rate of wound healing of burns and donor sites for skin grafts treated by FGF-RH was significantly higher than the placebo effect, and time to healing wounds of burns and donor sites for skin grafts the group of rh-aFGF was significantly shorter than in the placebo group. We conclude that topical administration of rh-aFGF can speed up the process of wound healing and shorten healing time. Therefore, it is a potential therapeutic application to promote the healing of deep partial thickness burns or donor sites for skin grafts [23].

Another study in 2008 evaluated the safety/tolerability of the recombinant human vascular endothelial growth factor (rh-VEGF; named telbermin) applied topically for chronic neuropathic foot ulcers diabetic. Topical application of $72 \mathrm{~cm}^{2}$ telbermin three times per week for up to six weeks appeared to be well tolerated. However, more studies are needed to more fully characterize the safety/efficacy of telbermin [50].

TGF-beta 3 (avotermin) was investigated as a possible potential anti-scarring therapy. The study was doubleblind, placebo-controlled, with intradermal injections avotermin. The results showed that avotermin has the potential to provide a rapid and permanent improvement in scarring [51].

Another study was conducted to evaluate the efficacy and safety of recombinant human epidermal growth factor (rh-EGF) on the healing of diabetic foot ulcers in patients. A total of 28 patients with foot ulcers were recruited for the pilot study. The treatment with this growth factor has positive effects on the healing of foot ulcers of moderate to severe condition and has been proven safe for diabetic patients. The drug had high tolerability and compliance [52].

It has been shown that bFGF promoted wound healing. Clinical efficacy and dose-response of bFGF in diabetic ulcer, a kind of refractory skin ulcers was evaluated. This was designed as a double-blind placebo-controlled randomized, dose-response. The results of this study showed effects of bFGF in accelerating wound healing in diabetic ulcers [53].

The cutaneous scar is associated with psychosocial distress and has a negative effect on the quality of life. The TGF-beta, the family of cytokines plays a key role in scar formation. TGF-beta 3 improves scar appearance in a range of mammalian species. This study was conducted to evaluate the efficacy of intradermal avotermin (TGF-beta 3) for improving the appearance of scarring after surgery to correct this condition. The primary data of the combined surgical avotermin groups showed significantly improved the appearance of scarring compared to placebo. The profilometry demonstrated a greater reduction in surface area from baseline to avotermin treatment compared with placebo scars. The administration of avotermin presented well tolerated and significantly improve the appearance of scarring compared to placebo [54].

A prospective, randomized, and phase II study was also investigated the avotermin as a reductor of scarring in the skin resulting in acute wound incisions. The results confirmed that avotermin, a compound of the growth factor, is the first of a new class of medicines that reduce the regenerative healing when administered once or twice in the next acute skin incisions [55].

The scar is a big problem of skin lesion. In addition, in another clinical study TGF-beta 3 (avotermin) also improved the appearance of the scar. This study aimed to determine whether the injection of avotermin at the time of wound closure is effective in improving the appearance of the scar. This was a double blind, randomized, patient, placebo-controlled study investigating the efficacy and safety of four doses of avotermin given at once. Avotermin $500 \mathrm{ng} / 100 \mathrm{ul}$ per linear cm wound margin in the given time is well tolerated and significantly improves scar appearance [56].

Pediatric burns present unique challenges. Second-degree burns may increase in size and depth, raised concerns about the healing and long-term healing. The results of a clinical study in adults with burn wounds from high school suggest that the application of bFGF can reduce the healing time and result in a more cosmetically acceptable scar. The effect of fibroblast growth factor has been reported in pediatric patients with burn wounds of deep second degree. The results showed that both the short and long-term results of this treatment in pediatric burn patients are encouraging and warrant further research [57]. BFGF has been clinically tested in burn injuries or surgery. The results showed improvement in all parameters in the scar [22].

Dressings of the biomaterial silk protein containing EGF were studied with a skin wound model in rats. All silk biomaterials were effective for wound healing. This systematic approach to assessing biomaterial dressings Functionalized silk demonstrates a useful strategy to select formulations for further study for new treatment options for chronic wounds [58]. 


\section{Conclusions}

With a better understanding of the phases of wound healing and action of growth factors in normal tissue without injury, new therapeutic modalities are being investigated for the treatment of problem wounds. Therefore, it is vital to realize that a number of growth factors and cytokines and inflammatory cells [13] govern the inflammation and wound healing.

Currently wound healing has many limitations, and this observation leads us to think further research to elaborate the development of safe, effective and economical way of therapies and treatments that improve the healing of wounds associated with various diseases in the population. Effective therapy would ideally reduce the bacterial load, establish control of severe inflammation, and concomitantly increase the tensile strength of the wound.

Therefore, it justifies the use of some growth factors, such as bFGF, TGF-beta 3, EGF and VEGF demonstrated here as effective in improving wound healing. However, many other growth factors (PDGF, TNF, KGF, IGF-I, IGF-II and NGF) seem to have been first proven in preclinical research and its clinical efficacy has yet to be validated in humans.

Therefore, we conclude that there is still a need for more clinical trials phase I and II in humans to better understand the role of other growth factors mentioned above, which have proven effective in preclinical trials, the improved healing of problematic wounds.

\section{Acknowledgements}

To Sao Paulo Research Foundation (FAPESP) and Coordination of Superior Level Staff Improvement (CAPES).

\section{References}

[1] Rojas, Y., Finnerty, C.C., Radhakrishnan, R.S. and Herndon, D.N. (2012) Burns: An Update on Current Pharmacotherapy. Expert Opinion on Pharmacotherapy, 13, 2485-2494. http://dx.doi.org/10.1517/14656566.2012.738195

[2] Zhao, G., Usui, M.L., Lippman, S.I., James, G.A., Stewart, P.S., Fleckman, P. and Olerud, J.E. (2013) Biofilms and Inflammation in Chronic Wounds. Advances in Wound Care (New Rochelle), 2, 389-399. http://dx.doi.org/10.1089/wound.2012.0381

[3] Sinno, H. and Prakash, S. (2013) Complements and the Wound Healing Cascade: An Updated Review. Plastic Surgery International, 2013, Article ID: 146764, 7 p.

[4] Sánchez, M., Anitua, E., Orive, G., Mujika, I. and Andia, I. (2009) Platelet-Rich Therapies in the Treatment of Orthopaedic Sport Injuries. Sports Medicine, 39, 345-354. http://dx.doi.org/10.2165/00007256-200939050-00002

[5] Kuebler, U., Wirtz, P.H., Sakai, M., Stemmer, A. and Ehlert, U. (2013) Acute Stress Reduces Wound-Induced Activation of Microbicidal Potential of ex Vivo Isolated Human Monocyte-Derived Macrophages. PLoS One, 8, e55875. http://dx.doi.org/10.1371/journal.pone.0055875

[6] Peplow, P.V. and Chatterjee, M.P. (2013) A Review of the Influence of Growth Factors and Cytokines in in Vitro Human Keratinocyte Migration. Cytokine, 62, 1-21. http://dx.doi.org/10.1016/j.cyto.2013.02.015

[7] Uutela, M., Wirzenius, M., Paavonen, K., Rajantie, I., He, Y., Karpanen, T., Lohela, M., Wiig, H., Salven, P., Pajusola, K., Eriksson, U. and Alitalo, K. (2004) PDGF-D Induces Macrophage Recruitment, Increased Interstitial Pressure, and Blood Vessel Maturation during Angiogenesis. Blood, 104, 3198-3204. http://dx.doi.org/10.1182/blood-2004-04-1485

[8] Hoeben, A., Landuyt, B., Highley, M.S., Wildiers, H., Van Oosterom, A.T. and De Bruijn, E.A. (2004) Vascular Endothelial Growth Factor and Angiogenesis. Pharmacological Reviews, 56, 549-580. http://dx.doi.org/10.1124/pr.56.4.3

[9] Yoo, S.Y. and Kwon, S.M. (2013) Angiogenesis and Its Therapeutic Opportunities. Mediators of Inflammation, 2013, Article ID 127170, $11 \mathrm{p}$.

[10] Eppley, B.L., Woodell, J.E. and Higgins, J. (2004) Platelet Quantification and Growth Factor Analysis from Platelet-Rich Plasma: Implications for Wound Healing. Plastic and Reconstructive Surgery, 114, 1502-1508. http://dx.doi.org/10.1097/01.PRS.0000138251.07040.51

[11] Riedel, K., Riedel, F., Goessler, U.R., Germann, G. and Sauerbier, M. (2007) TGF- $\beta$ Antisense Therapy Increases Angiogenic Potential in Human Keratinocytes in Vitro. Archives of Medical Research, 38, 45-51. http://dx.doi.org/10.1016/j.arcmed.2006.04.010

[12] Broughton II, G., Janis, J.E. and Attinger, C.E. (2006) The Basic Science of Wound Healing. Plastic \& Reconstructive Surgery, 117, 12S-34S. http://dx.doi.org/10.1097/01.prs.0000225430.42531.c2

[13] Chambers, R.C. (2008) Procoagulant Signalling Mechanisms in Lung Inflammation and Fibrosis: Novel Opportunities 
for Pharmacological Intervention? British Journal of Pharmacology, 153, S367-S378. http://dx.doi.org/10.1038/sj.bjp.0707603

[14] Verhaegen, P.D., van Zuijlen, P.P., Pennings, N.M., van Marle, J., Niessen, F.B., van der Horst, C.M. and Middelkoop, E. (2009) Differences in Collagen Architecture between Keloid, Hypertrophic Scar, Normotrophic Scar, and Normal Skin: An Objective Histopathological Analysis. Wound Repair and Regeneration, 17, 649-656. http://dx.doi.org/10.1111/j.1524-475X.2009.00533.x

[15] Greenhalgh, D.G. (2005) Models of Wound Healing. Journal of Burn Care \& Rehabilitation, 26, 293-305. http://dx.doi.org/10.1097/01.BCR.0000169885.66639.B5

[16] Vande Berg, J.S., Rose, M.A., Haywood-Reid, P.L., Rudolph, R., Payne, W.G. and Robson, M.C. (2005) Cultured Pressure ulcer Fibroblasts Show Replicative Senescence with Elevated Production of Plasmin, Plasminogen Activator Inhibitor-1, and Transforming Growth Factor-Beta1. Wound Repair and Regeneration, 13, 76-83. http://dx.doi.org/10.1111/j.1067-1927.2005.130110.x

[17] Aspenberg, P. and Virchenko, O. (2004) Platelet Concentrate Injection Improves Achilles Tendon Repair in Rats. Acta Orthopaedica, 75, 93-99. http://dx.doi.org/10.1080/00016470410001708190

[18] Mishra, A., Harmon, K., Woodall, J. and Vieira, A. (2011) Sports Medicine Applications of Platelet Rich Plasma. Current Pharmaceutical Biotechnology, 13, 1185-1195. http://dx.doi.org/10.2174/138920112800624283

[19] Boo, S. and Dagnino, L. (2013) Integrins as Modulators of Transforming Growth Factor Beta Signaling in Dermal Fibroblasts during Skin Regeneration after Injury. Advances in Wound Care (New Rochelle), 2, 238-246. http://dx.doi.org/10.1089/wound.2012.0394

[20] Zheng, Z., Kang, H.Y., Lee, S., Kang, S.W., Goo, B. and Cho, S.B. (2014) Upregulation of Fibroblast Growth Factor (FGF) 9 Expression and FGF-WNT/ $\beta$-Catenin Signaling in Laser-Inducedwound Healing. Wound Repair and Regeneration, 22, 660-665. http://dx.doi.org/10.1111/wrr.12212

[21] Chrissouli, S., Pratsinis, H., Velissariou, V., Anastasiou, A. and Kletsas, D. (2010) Human Amniotic Fluid Stimulates the Proliferation of Human Fetal and Adult Skin Fibroblasts: The Roles of bFGF and PDGF and of the ERK and Akt Signaling Pathways. Wound Repair and Regeneration, 18, 643-654. http://dx.doi.org/10.1111/j.1524-475X.2010.00626.x

[22] Akita, S., Akino, K., Imaizumi, T. and Hirano, A. (2008) Basic Fibroblast Growth Factor Accelerates and Improves Second-Degree Burn Wound Healing. Wound Repair and Regeneration, 16, 635-641. http://dx.doi.org/10.1111/j.1524-475X.2008.00414.X

[23] Ma, B., Cheng, D.S., Xia, Z.F., Ben, D.F., Lu, W., Cao, Z.F., et al. (2007) Randomized, Multicenter, Double-Blind, and Placebo-Controlled Trial Using Topical Recombinant Human Acidic Fibroblast Growth Factor for Deep PartialThickness Burns and Skin Graft Donor Site. Wound Repair and Regeneration, 15, 795-799. http://dx.doi.org/10.1111/j.1524-475X.2007.00307.x

[24] Bao, S., Wang, Y., Sweeney, P., Chaudhuri, A., Doseff, A.I., Marsh, C.B. and Knoell, D.L. (2005) Keratinocyte Growth Factor Induces Akt Kinase Activity and Inhibits Fas-Mediated Apoptosis in A549 Lung Epithelial Cells. American Journal of Physiology-Lung Cellular and Molecular Physiology, 288, L36-L42. http://dx.doi.org/10.1152/ajplung.00309.2003

[25] Koivisto, L., Jiang, G., Häkkinen, L., Chan, B. and Larjava, H. (2006) HaCaT Keratinocyte Migration Is Dependent on Epidermal Growth Factor Receptor Signaling and Glycogen Synthase Kinase-3 $\alpha$. Experimental Cell Research, 312, 2791-2805. http://dx.doi.org/10.1016/j.yexcr.2006.05.009

[26] Gomperts, B.N., Belperio, J.A., Fishbein, M.C., Keane, M.P., Burdick, M.D. and Strieter, R.M. (2007) Keratinocyte Growth Factor Improves Repair in the Injured Tracheal Epithelium. American Journal of Respiratory Cell and Molecular Biology, 37, 48-56. http://dx.doi.org/10.1165/rcmb.2006-0384OC

[27] Alemdaroglu, C., Degim, Z., Celebi, N., Zor, F., Ozturk, S. and Erdogan, D. (2006) An Investigation on Burn Wound Healing in Rats with Chitosan Gel Formulation Containing Epidermal Growth Factor. Burns, 32, 319-327. http://dx.doi.org/10.1016/j.burns.2005.10.015

[28] Alemdaroglu, C., Degim, Z., Celebi, N., Sengezer, M., Alomeroglu, M. and Nacar, A. (2008) Investigation of Epidermal Growth Factor Containing Liposome Formulation Effects on Burn Wound Healing. Journal of Biomedical Materials Research Part A, 85, 271-283. http://dx.doi.org/10.1002/jbm.a.31588

[29] Jahovic, N., Guzel, E., Arbak, S. and Yegen, B.C. (2004) The Healing-Promoting Effect of Saliva on Skin Burn Is Mediated by Epidermal Growth Factor (EGF): Role of the Neutrophils. Burns, 30, 531-538. http://dx.doi.org/10.1016/j.burns.2004.02.007

[30] Barrientos, S., Stojadinovic, O., Golinko, M.S., Brem, H. and Tomic-Canic, M. (2008) Growth Factors and Cytokines in Wound Healing. Wound Repair and Regeneration, 16, 585-601. http://dx.doi.org/10.1111/j.1524-475X.2008.00410.x 
[31] Infanger, M., Schmidt, O., Kossmehl, P., Grad, S., Ertel, W. and Grimm, D. (2004) Vascular Endothelial Growth Factor Serum Level Is Strongly Enhanced after Burn Injury and Correlated with Local and General Tissue Edema. Burns, 30, 305-311. http://dx.doi.org/10.1016/j.burns.2003.12.006

[32] Bao, P., Kodra, A., Tomic-Canic, M., Golinko, M.S., Ehrlich, H.P. and Brem, H. (2009) The Role of Vascular Endothelial Growth Factor in Wound Healing. Journal of Surgical Research, 153, 347-358. http://dx.doi.org/10.1016/j.jss.2008.04.023

[33] Garovic, V.D., Wagner, S.J., Petrovic, L.M., Gray, C.E., Hall, P., Sugimoto, H., et al. (2007) Glomerular Expression of Nephrin and Synaptopodin, but Not Podocin, Is Decreased in Kidney Sections from Women with Preeclampsia. Nephrology Dialysis Transplantation, 22, 1136-1143. http://dx.doi.org/10.1093/ndt/gfl711

[34] Nagy, J.A., Chang, S.H., Shih, S.C., Dvorak, A.M. and Dvorak, H.F. (2010) Heterogeneity of the Tumor Vasculature. Seminars in Thrombosis and Hemostasis, 36, 321-331. http://dx.doi.org/10.1055/s-0030-1253454

[35] Eming, S.A. and Krieg, T. (2006) Molecular Mechanisms of VEGF-A Action during Tissue Repair. Journal of Investigative Dermatology Symposium Proceedings, 11, 79-86. http://dx.doi.org/10.1038/sj.jidsymp.5650016

[36] Shen, F., Su, H., Fan, Y., Chen, Y., Zhu, Y., Liu, W., Young, W.L. and Yang, G.Y. (2006) Adeno-Associated ViralVector-Mediated Hypoxia-Inducible Vascular Endothelial Growth Factor Gene Expression Attenuates Ischemic Brain Injury after Focal Cerebral Ischemia in Mice. Stroke, 37, 2601-2606. http://dx.doi.org/10.1161/01.STR.0000240407.14765.e8

[37] Chen, J.C., Lin, B.B., Hu, H.W., Lin, C., Jin, W.Y., Zhang, F.B., Zhu, Y.A., Lu, C.J., Wei, X.J. and Chen, R.J. (2014) NGF Accelerates Cutaneous Wound Healing by Promoting the Migration of Dermal Fibroblasts via the PI3K/AktRac1-JNK and ERK Pathways. BioMed Research International, 2014, Article ID: 547187.

[38] Palazzo, E., Marconi, A., Truzzi, F., Dallaglio, K., Petrachi, T., Humbert, P., Schnebert, S., Perrier, E., Dumas, M. and Pincelli, C. (2012) Role of Neurotrophins on Dermal Fibroblast Survival and Differentiation. Journal of Cellular Physiology, 227, 1017-1025. http://dx.doi.org/10.1002/jcp.22811

[39] Pakyari, M., Farrokhi, A., Maharlooei, M.K. and Ghahary, A. (2013) Critical Role of Transforming Growth Factor Beta in Different Phases of Wound Healing. Advances in Wound Care (New Rochelle), 2, 215-224. http://dx.doi.org/10.1089/wound.2012.0406

[40] Finnson, K.W., Arany, P.R. and Philip, A. (2013) Transforming Growth Factor Beta Signaling in Cutaneous Wound Healing: Lessons Learned from Animal Studies. Advances in Wound Care (New Rochelle), 2, 225-237. http://dx.doi.org/10.1089/wound.2012.0419

[41] Denis, J.F., Lévesque, M., Tran, S.D., Camarda, A.J. and Roy, S. (2013) Axolotl as a Model to Study Scarless Wound Healing in Vertebrates: Role of the Transforming Growth Factor Beta Signaling Pathway. Advances in Wound Care (New Rochelle), 2, 250-260. http://dx.doi.org/10.1089/wound.2012.0371

[42] Chen, W., Fu, X., Ge, S., Sun, T.Z., Zhou, G., Jiang, D.Y. and Sheng, Z.Y. (2005) Ontogeny of Expression of Transforming Growth Factor-Beta and Its Receptors and Their Possible Relationship with Scarless Healing in Human Fetal Skin. Wound Repair and Regeneration, 13, 68-75. http://dx.doi.org/10.1111/j.1067-1927.2005.130109.x

[43] Durani, P., Occleston, N., O’Kane, S. and Ferguson, M.W. (2008) Avotermin: A Novel Antiscarring Agent. International Journal of Lower Extremity Wounds, 7, 160-168. http://dx.doi.org/10.1177/1534734608322983

[44] Finnson, K.W., McLean, S., Di Guglielmo, G.M. and Philip, A. (2013) Dynamics of Transforming Growth Factor Beta Signaling in Wound Healing and Scarring. Advances in Wound Care (New Rochelle), 2, 195-214. http://dx.doi.org/10.1089/wound.2013.0429

[45] Singer, A.J., Huang, S.S., Huang, J.S., McClain, S.A., Romanov, A., Rooney, J. and Zimmerman, T. (2009) A Novel TGF-Beta Antagonist Speeds Reepithelialization and Reduces Scarring of Partial Thickness Porcine Burns. Journal of Burn Care \& Research, 30, 329-334. http://dx.doi.org/10.1097/BCR.0b013e31819a6369

[46] Occleston, N.L., Laverty, H.G., O’Kane, S. and Ferguson, M.W. (2008) Prevention and Reduction of Scarring in the Skin by Transforming Growth Factor $\beta 3$ (TGF $\beta 3$ ): From Laboratory Discovery to Clinical Pharmaceutical. Journal of Biomaterials Science, Polymer Edition, 19, 1047-1063. http://dx.doi.org/10.1163/156856208784909345

[47] Mann, A., Niekisch, K., Schirmacher, P. and Blessing, M. (2006) Granulocyte-Macrophage Colony-Stimulating Factor Is Essential for Normal Wound Healing. Journal of Investigative Dermatology Symposium Proceedings, 11, 87-92. http://dx.doi.org/10.1038/sj.jidsymp.5650013

[48] Hu, X., Sun, H., Han, C.M., Wang, X.G. and Yu, W.J. (2011) Topically Applied rhGM-CSF for the Wound Healing: A Systematic Review. Burns, 37, 729-741. http://dx.doi.org/10.1016/j.burns.2010.08.016

[49] Yao, C., Yao, P., Wu, H. and Zha, Z.G. (2006) Acceleration of Wound Healing in Traumatic Ulcers by Absorbable Collagen Sponge Containing Recombinant Basic Fibroblast Growth Factor. Biomedical Materials, 1, 33-37. http://dx.doi.org/10.1088/1748-6041/1/1/005

[50] Hanft, J.R., Pollak, R.A., Barbul, A., van Gils, C., Kwon, P.S., Gray, S.M., Lynch, C.J., Semba, C.P. and Breen, T.J. 
(2008) Phase I Trial on the Safety of Topical rhVEGF on Chronic Neuropathic Diabetic Foot Ulcers. Journal of Wound Care, 17, 30-32, 34-37. http://dx.doi.org/10.12968/jowc.2008.17.1.27917

[51] Ferguson, M.W., Duncan, J., Bond, J., Bush, J., Durani, P., So, K., Taylor, L., Chantrey, J., Mason, T., James, G., Laverty, H., Occleston, N.L., Sattar, A., Ludlow, A. and O’Kane, S. (2009) Prophylactic Administration of Avotermin for Improvement of Skin Scarring: Three Double-Blind, Placebo-Controlled, Phase I/II Studies. Lancet, 373, 12641274. http://dx.doi.org/10.1016/S0140-6736(09)60322-6

[52] Tuyet, H.L., Nguyen Quynh, T.T., Vo Hoang Minh, H., Thi Bich, D.N., Do Dinh, T., Le Tan, D., Van, H.L., Le Huy, T., Doan Huu, H. and Tran Trong, T.N. (2009) The Efficacy and Safety of Epidermal Growth Factor in Treatment of Diabetic Foot Ulcers: The Preliminary Results. International Wound Journal, 6, 159-166. http://dx.doi.org/10.1111/j.1742-481X.2009.00594.x

[53] Uchi, H., Igarashi, A., Urabe, K., Koga, T., Nakayama, J., Kawamori, R., Tamaki, K., Hirakata, H., Ohura, T. and Furue, M. (2009) Clinical Efficacy of Basic Fibroblast Growth Factor (bFGF) for Diabetic Ulcer. European Journal of Dermatology, 19, 461-468.

[54] So, K., McGrouther, D.A., Bush, J.A., Durani, P., Taylor, L., Skotny, G., Mason, T., Metcalfe, A., O’kane, S. and Ferguson, M.W. (2011) Avotermin for Scar Improvement Following Scar Revision Surgery: A Randomized, DoubleBlind, Within-Patient, Placebo-Controlled, Phase II Clinical Trial. Plastic \& Reconstructive Surgery, 128, $163-172$. http://dx.doi.org/10.1097/PRS.0b013e318217429b

[55] Bush, J., Duncan, J.A., Bond, J.S., Durani, P., So, K., Mason, T., O’Kane, S. and Ferguson, M.W. (2010) Scar-Improving Efficacy of Avotermin Administered into the Wound Margins of Skin Incisions as Evaluated by a Randomized, Double-Blind, Placebo-Controlled, Phase II Clinical Trial. Plastic \& Reconstructive Surgery, 126, 1604-1615. http://dx.doi.org/10.1097/PRS.0b013e3181ef8e66

[56] McCollum, P.T., Bush, J.A., James, G., Mason, T., O’Kane, S., McCollum, C., Krievins, D., Shiralkar, S. and Ferguson, M.W. (2011) Randomized Phase II Clinical Trial of Avotermin versus Placebo for Scar Improvement. British Journal of Surgery, 98, 925-934. http://dx.doi.org/10.1002/bjs.7438

[57] Hayashida, K. and Akita, S. (2012) Quality of Pediatric Second-Degree Burn Wound Scars Following the Application of Basic Fibroblast Growth Factor: Results of a Randomized, Controlled Pilot Study. Ostomy/Wound Management, 58, 32-36.

[58] Gil, E.S., Panilaitis, B., Bellas, E. and Kaplan, D.L. (2013) Functionalized Silk Biomaterials for Wound Healing. Advanced Healthcare Materials, 2, 206-217. http://dx.doi.org/10.1002/adhm.201200192 
Scientific Research Publishing (SCIRP) is one of the largest Open Access journal publishers. It is currently publishing more than 200 open access, online, peer-reviewed journals covering a wide range of academic disciplines. SCIRP serves the worldwide academic communities and contributes to the progress and application of science with its publication.

Other selected journals from SCIRP are listed as below. Submit your manuscript to us via either submit@scirp.org or Online Submission Portal.
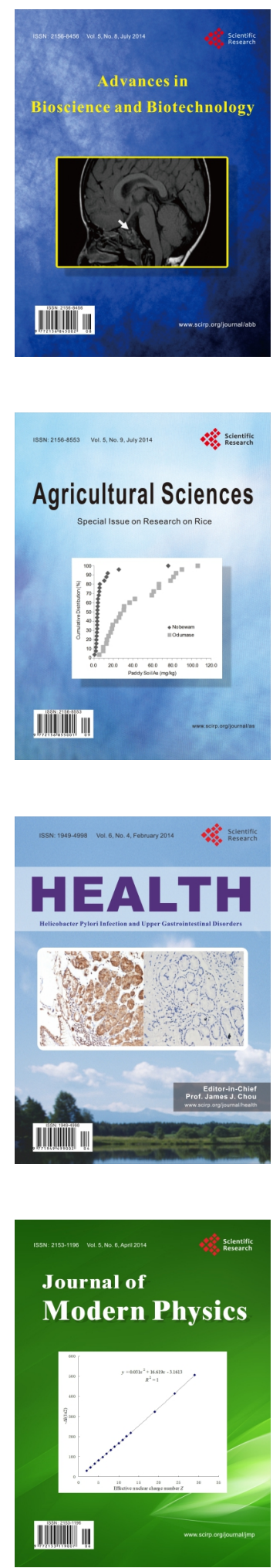
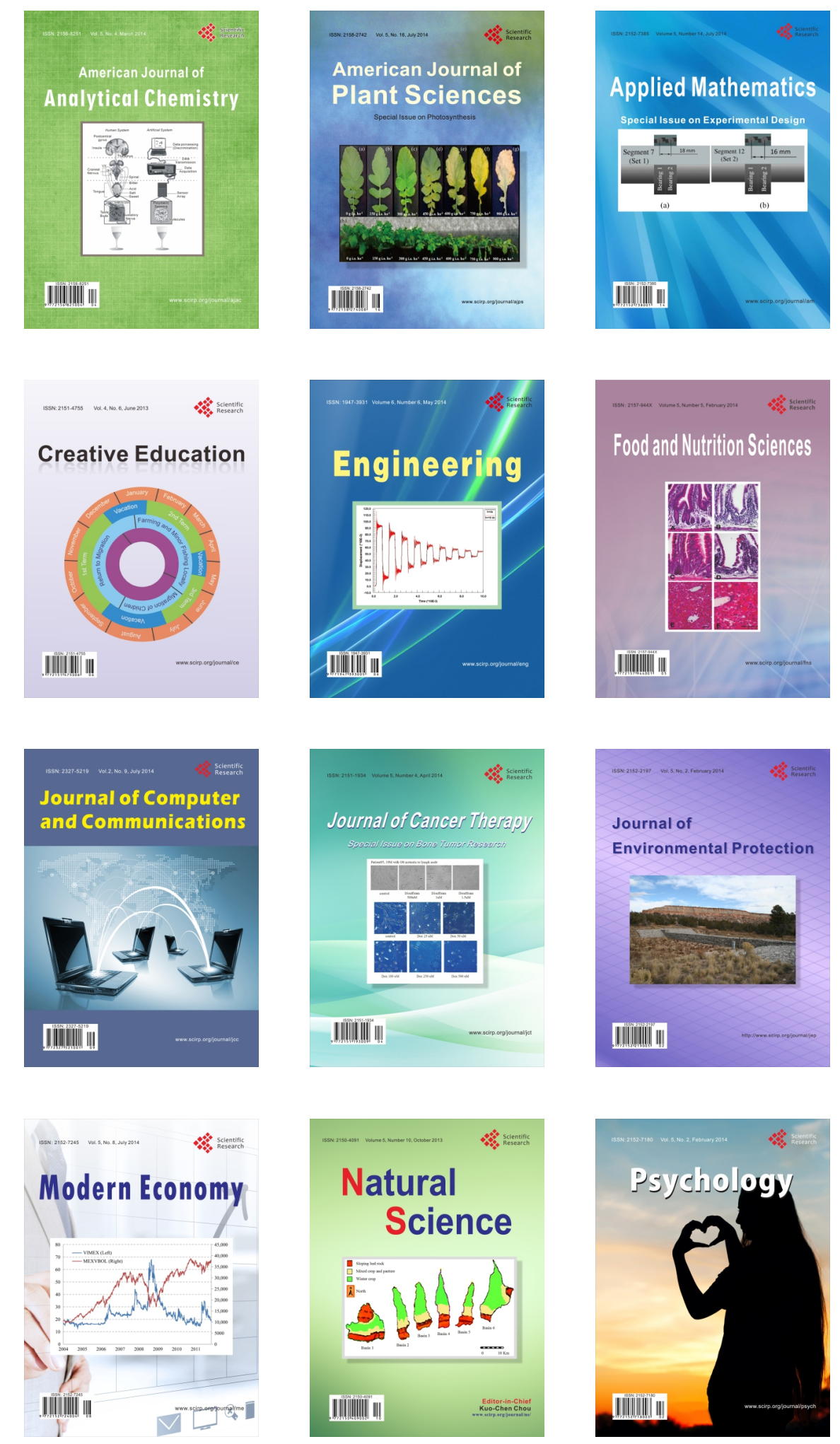